\title{
Remarks on Liouville type theorems for the 3D steady axially symmetric Navier-Stokes equations \\ Wendong Wang
}

Dalian University of Technology, China

\&University of Oxford, UK

April 29, 2019

\begin{abstract}
In this note, we investigate the 3D steady axially symmetric Navier-Stokes equations, and obtained Liouville type theorems if the velocity or the vorticity satisfies some a priori decay assumptions.
\end{abstract}

Keywords: Liouville type theorem, Navier-Stokes equations, axially symmetric NavierStokes equations

\section{Introduction}

An interesting question about Liouville type theorem of the 3D stationary Navier-Stokes equations in $R^{3}$ is as follows: whether the solution of

$$
\left\{\begin{array}{l}
-\Delta u+u \cdot \nabla u=-\nabla p, \\
\nabla \cdot u=0,
\end{array}\right.
$$

satisfying the vanishing property at infinity

$$
\lim _{|x| \rightarrow \infty} u(x)=0
$$

and the bounded Dirichlet energy

$$
D(u)=\int_{R^{3}}|\nabla u|^{2} d x<\infty
$$


implies $u \equiv 0$ is still an open problem, which is related to J. Leray (see also P12. Galdi $[7])$.

Many conditional criteria have been obtained for this issue. For example, Galdi proved the above Liouville type theorem by assuming $u \in L^{\frac{9}{2}}\left(R^{3}\right)$ in [7]. Chae in [2] showed the condition $\triangle u \in L^{\frac{6}{5}}\left(R^{3}\right)$ is sufficient for the vanishing property of $u$. Also, Chae-Wolf gave an improvement of logarithmic form for Galdi's result in [4] by assuming that $\int_{R^{3}}|u|^{\frac{9}{2}}\left\{\ln \left(2+\frac{1}{|u|}\right)\right\}^{-1} d x<\infty$. Seregin obtained the conditional criterion $u \in B M O^{-1}\left(R^{3}\right)$ in [12]. Moreover, Kozonoa-Terasawab-Wakasugib proved $u \equiv 0$ if the vorticity $w=o\left(|x|^{-\frac{5}{3}}\right)$ or $\|u\|_{L^{\frac{9}{2}, \infty}\left(R^{3}\right)} \leq \delta D(u)^{1 / 3}$ for a small constant $\delta$ in [10]. It is shown that all the above norms $u \in L^{\frac{9}{2}}\left(R^{3}\right)$, the log form of $u \in L^{\frac{9}{2}}\left(R^{3}\right)$ or $u \in L^{\frac{9}{2}, \infty}\left(R^{3}\right)$ can be replaced by the norms in the annular domain $B_{R} \backslash B_{R / 2}$ in [16] by Seregin and the author, where the following energy description was stated:

$$
\int_{B_{R / 2}}|\nabla u|^{2} d x \leq C R^{-2}\left(\int_{B_{R} \backslash B_{R / 2}}|u|^{2} d x\right)+C(q) R^{2-\frac{9}{q}}\|u\|_{L^{q, \infty}\left(B_{R} \backslash B_{R / 2}\right)}^{3}
$$

where $B_{R}=B_{R}(0)$ is a ball centered at 0 and $q>3$. Note that the conditions (2) and (3) are not used in [16] as in [4]. More references, we refer to [3, 13, 14] and the references therein.

Moreover, the problem is not solved, even for the case of axially symmetric NavierStokes equations, to the best of the author's knowledge. Motivated by the result Seregin in [14], where he proved that the condition $|u| \lesssim \frac{1}{\left|x^{\prime}\right|^{\mu}}$ with $x^{\prime}=\left(x_{1}, x_{2}\right)$ and $\mu \approx 0.77$ implies $u \equiv 0$, we are aimed to improve the decay assumption. At first, let us introduce the axially symmetric Navier-Stokes equations. Let $u(x)=u_{r}(t, r, z) e_{r}+u_{\theta}(t, r, z) e_{\theta}+u_{z}(t, r, z) e_{z}$, where

$$
\begin{aligned}
e_{r} & =\left(\frac{x_{1}}{r}, \frac{x_{2}}{r}, 0\right)=(\cos \theta, \sin \theta, 0), \\
e_{\theta} & =\left(-\frac{x_{2}}{r}, \frac{x_{1}}{r}, 0\right)=(-\sin \theta, \cos \theta, 0), \\
e_{z} & =(0,0,1)
\end{aligned}
$$

and (1) becomes

$$
\left\{\begin{array}{l}
b \cdot \nabla u_{r}-\Delta_{0} u_{r}+\frac{u_{r}}{r^{2}}-\frac{u_{\theta}^{2}}{r}+\partial_{r} p=0 \\
b \cdot \nabla u_{\theta}-\Delta_{0} u_{\theta}+\frac{u_{\theta}}{r^{2}}+\frac{u_{r} u_{\theta}}{r}=0 \\
b \cdot \nabla u_{z}-\Delta_{0} u_{z}+\partial_{z} p=0 \\
\partial_{r}\left(r u_{r}\right)+\partial_{z}\left(r u_{z}\right)=0
\end{array}\right.
$$

where

$$
b=u_{r} e_{r}+u_{z} e_{z}, \quad \Delta_{0}=\partial_{r r}+\frac{1}{r} \partial_{r}+\partial_{z z} .
$$


The vorticity is represented as

$$
w=w_{r} e_{r}+w_{\theta} e_{\theta}+w_{z} e_{z}=\left(-\partial_{z} u_{\theta}\right) e_{r}+\left(\partial_{z} u_{r}-\partial_{r} u_{z}\right) e_{\theta}+\frac{\partial_{r}\left(r u_{\theta}\right)}{r} e_{z} .
$$

There are also many developments on the Liouville type theorems of axi-symmetric case. For example, Liouville type theorem was proved by assuming no swirl (i.e. $u_{\theta}=0$ ), see Koch-Nadirashvili-Seregin-Sverak [9] or Korobkov-Pileckas-Russo[11]. The condition $r u_{\theta} \in L^{q}$ with some $q \geq 1$ or $b \in L^{3}$ is enough, see Chae-Weng in [5]. Specially, for the axially symmetric case, the decay of the velocity or the vorticity can be obtained: Choe-Jin [6], Weng [17] proved that

$$
\begin{aligned}
& \left|u_{r}(r, z)\right|+\left|u_{z}(r, z)\right|+\left|u_{\theta}(r, z)\right| \lesssim \sqrt{\frac{\ln r}{r}} \\
& \left|w_{\theta}(r, z)\right| \lesssim r^{-\left(\frac{19}{16}\right)^{-}}, \quad\left|w_{r}(r, z)\right|+\left|w_{z}(r, z)\right| \lesssim r^{-\left(\frac{17}{16}\right)^{-}}
\end{aligned}
$$

Recently, Carrillo-Pan-Zhang in [1] gave an alternative method for the decay of $u$ and an improvement for the decay bound of the vorticity

$$
\left|w_{\theta}(r, z)\right| \lesssim r^{-\frac{5}{4}}(\ln r)^{\frac{3}{4}}, \quad\left|w_{r}(r, z)\right|+\left|w_{z}(r, z)\right| \lesssim r^{-\frac{9}{8}}(\ln r)^{\frac{11}{8}}
$$

by using Brezis-Gallouet inequality.

It's a natural question: whether there exist the sharp constants $\mu_{1}, \mu_{2}$ such that $\left|\left(u_{r}(r, z), u_{z}(r, z), u_{\theta}(r, z)\right)\right| \lesssim \frac{1}{r^{\mu_{1}}}$ or $\left|\left(w_{r}(r, z), w_{z}(r, z), w_{\theta}(r, z)\right)\right| \lesssim \frac{1}{r^{\mu_{2}}}$ implies that $u \equiv 0$ for the axially symmetric case?

With the help of energy estimates in [16] we can improve the result in [14] to $\mu>\frac{2}{3}$, which is almost a equivalent form of $u \in L^{\frac{9}{2}, \infty}$.

Theorem 1.1. Suppose that $u$ is axially symmetric smooth solution of the equation (4) and for some $\mu>\frac{2}{3}$,

$$
|u| \leq \frac{C}{(1+r)^{\mu}}
$$

Then $u \equiv 0$.

Note that $\Gamma=r u_{\theta}$ satisfies the special structure

$$
b \cdot \nabla \Gamma-\triangle_{0} \Gamma+\frac{2}{r} \partial_{r} \Gamma=0
$$

and Maximum principle can be applied, thus the condition $u_{\theta}=o\left(\frac{1}{r}\right)$ as $|x| \rightarrow \infty$ implies $u$ is trivial. However, it's still known that whether $u_{\theta}=o\left(\frac{1}{r}\right)$ can be replaced by $u_{\theta}=O\left(\frac{1}{r}\right)$. But we show that the condition $|b|=O\left(\frac{1}{r}\right)$ or $b \in B M O^{-1}\left(R^{3}\right)$ is sufficient, which improved the assumption $b \in L^{3}\left(R^{3}\right)$ in [5]. 
Here we say a function $f \in B M O^{-1}\left(R^{3}\right)$ if there exists a vector-value function $d \in R^{3}$ and $d_{j} \in B M O\left(R^{3}\right)$ such that $f=d i v d=d_{j, j}$. It's well-known that for the BMO space, we have

$$
\Gamma(s)=\sup _{x_{0} \in R^{3}, R>0}\left(\frac{1}{\left|B_{R}\left(x_{0}\right)\right|} \int_{B_{R}\left(x_{0}\right)}\left|d-d_{x_{0}, R}\right|^{s} d x\right)^{\frac{1}{s}}<\infty .
$$

for any $s \in[1, \infty)$.

In details, we obtained the following result.

Theorem 1.2. Suppose that $u$ is axially symmetric smooth solution of the equation (4) satisfying (2) and (3). Then $u \equiv 0$ if one of the following conditions is satisfied

$$
\begin{aligned}
& \text { (i) } b=\left(u_{r}, u_{z}\right) \in B M O^{-1}\left(R^{3}\right) ; \\
& \text { (ii) }|b| \leq \frac{C}{r}
\end{aligned}
$$

For the decay of the vorticity, we also state the following corresponding result.

Theorem 1.3. Suppose that $u$ is axially symmetric smooth solution of the equation (4) satisfying (2) and (3). Moreover,

$$
\left|\left(w_{r}, w_{\theta}, w_{z}\right)\right| \leq \frac{C}{r^{\beta}}, \quad \beta>\frac{5}{3}
$$

Then $u \equiv 0$.

Remark 1. This conclusion generalized the result of [10] to the axially symmetric case, where the condition $|w|=o\left(|x|^{-\frac{5}{3}}\right)$ was put.

Throughout this article, $C$ denotes a constant, which may be different from line to line.

\section{Proof of Theorem 1.1}

Recall a Caccioppoli inequality in [16], which is stated as follows.

Proposition 2.1. Let $(u, p)$ be the smooth solution of (1). Then for $0<\delta \leq 1$ and $\frac{6(3-\delta)}{6-\delta}<q<3$, we have

$$
\begin{aligned}
\int_{B_{R / 2}}|\nabla u|^{2} d x \leq & \frac{C}{R^{2}}\left(\int_{B_{R} \backslash B_{R / 2}}|u|^{2} d x\right) \\
& +C(\delta)\left(\|u\|_{L^{q, \infty}\left(B_{R} \backslash B_{R / 2}\right)}^{3-\delta} R^{2-\frac{9-3 \delta}{q}-\frac{\delta}{2}}\right)^{\frac{2}{2-\delta}}
\end{aligned}
$$


Proof of Theorem 1.1. Let $C_{R}$ denote the cylindrical region $\left\{x ;\left|x^{\prime}\right| \leq R,|z| \leq R\right\}$, then it's easy to check that

$$
B_{R} \subset C_{R} \subset B_{\sqrt{2} R} .
$$

Hence, it follows from Proposition 2.1 that

$$
\begin{aligned}
\int_{C_{\frac{\sqrt{2}}{4} R}}|\nabla u|^{2} d x \leq & \frac{C}{R^{2}}\left(\int_{C_{R} \backslash C_{\frac{\sqrt{2}}{4} R}}|u|^{2} d x\right) \\
& +C(\delta)\left(\|u\|_{L^{q, \infty}\left(C_{R} \backslash C_{\frac{\sqrt{2}}{4} R}\right.}^{3-\delta} R^{2-\frac{9-3 \delta}{q}-\frac{\delta}{2}}\right)^{\frac{2}{2-\delta}} \\
\leq & C\|u\|_{L^{q}\left(C_{R}\right)}^{2} R^{1-\frac{6}{q}}+C(\delta, q)\left(\|u\|_{L^{q}\left(C_{R}\right)}^{3-\delta} R^{2-\frac{9-3 \delta}{q}-\frac{\delta}{2}}\right)^{\frac{2}{2-\delta}}
\end{aligned}
$$

for $q>2$, where we used the property of Lorentz space

$$
\|u\|_{L^{q, \infty}(\Omega)} \leq C(q, \ell)\|u\|_{L^{q, \ell}(\Omega)}
$$

(for example, see Proposition 1.4.10 in [8]).

For $\mu q>2$, we have

$$
\|u\|_{L^{q}\left(C_{R}\right)} \leq C\left(R \int_{0}^{R}(1+r)^{1-\mu q} d r\right)^{\frac{1}{q}} \leq C(\mu, q) R^{\frac{1}{q}}
$$

Then the terms of the right hand side of (5) is controlled by

$$
\int_{C_{\frac{\sqrt{2}}{4} R}}|\nabla u|^{2} d x \leq C(\mu, q) R^{1-\frac{4}{q}}+C(\delta, \mu, q)\left(R^{2-\frac{\delta}{2}-\frac{6-2 \delta}{q}}\right)^{\frac{2}{2-\delta}}
$$

Claim that: for fixed $\mu>\frac{2}{3}$, there exist constants $\delta \in(0,1)$ and $q$ such that

$$
\max \left\{6 \frac{3-\delta}{6-\delta}, \frac{2}{\mu}\right\}<q<3, \quad \text { and } 2-\frac{\delta}{2}-\frac{6-2 \delta}{q}<0
$$

hence letting $R \rightarrow \infty$, by (6) we have

$$
\int_{R^{3}}|\nabla u|^{2} d x=0
$$

which implies $u \equiv 0$.

Proof of (7). First for fixed $\mu>\frac{2}{3}$, we choose $\delta_{0} \in(0,1)$ such that

$$
\frac{2}{\mu}<4 \frac{3-\delta_{0}}{4-\delta_{0}}
$$


Since $0<\delta_{0}<1$, we have

$$
1-\frac{\delta_{0}}{4}<1-\frac{\delta_{0}}{6}
$$

and

$$
6 \frac{3-\delta_{0}}{6-\delta_{0}}<4 \frac{3-\delta_{0}}{4-\delta_{0}}
$$

so we take

$$
q=\frac{1}{2}\left(\max \left\{6 \frac{3-\delta_{0}}{6-\delta_{0}}, \frac{2}{\mu}\right\}+4 \frac{3-\delta_{0}}{4-\delta_{0}}\right)
$$

Then we have

$$
\max \left\{6 \frac{3-\delta_{0}}{6-\delta_{0}}, \frac{2}{\mu}\right\}<q<4 \frac{3-\delta_{0}}{4-\delta_{0}}<3,
$$

which implies (7).

Hence the proof of Theorem 1.1 is complete.

\section{Proof of Theorem 1.2}

Let $\phi(x)=\phi(r, z) \in C_{0}^{\infty}\left(C_{R}\right)$ and $0 \leq \phi \leq 1$ satisfying

$$
\phi(x)= \begin{cases}1, & x \in C_{R / 2}, \\ 0, & x \in C_{R}^{c}\end{cases}
$$

and

$$
|\nabla \phi| \leq \frac{C}{R}, \quad\left|\nabla^{2} \phi\right| \leq \frac{C}{R^{2}} .
$$

Without loss of generality, by Theorem X.5.1 in [7] we can assume that

$$
\lim _{|x| \rightarrow \infty}|p|+|u|=0 .
$$

Note that $\triangle p=-\partial_{i} \partial_{j}\left(u_{i} u_{j}\right)$, then using Calderón-Zygmund estimates and gradient estimates of harmonic function, we have

$$
\int_{R^{3}}|p|^{3}+|u|^{6} d x<C D(u)^{3},
$$

and

$$
\|\nabla p\|_{L^{\frac{3}{2}\left(R^{3}\right)}}<C D(u),
$$


since $\||\nabla u| u\|_{L^{\frac{3}{2}\left(R^{3}\right)}} \leq C D(u)$.

Multiplying $\phi u \cdot$ on both sides of (1), integration by parts yields that

$$
\begin{aligned}
& \int_{C_{R}} \phi\left(\left|\nabla u_{r}\right|^{2}+\left|\nabla u_{\theta}\right|^{2}+\left|\nabla u_{z}\right|^{2}+\frac{u_{r}^{2}}{r^{2}}+\frac{u_{\theta}^{2}}{r^{2}}\right) d x \\
\leq & \int_{C_{R}}\left(\frac{1}{2}|u|^{2}+p\right)\left(u_{r} \partial_{r}+u_{z} \partial_{z}\right) \phi d x+C\|u\|_{L^{6}\left(C_{R} \backslash C_{R / 2}\right)}^{2} \\
\doteq & I+C\|u\|_{L^{6}\left(C_{R} \backslash C_{R / 2}\right)}^{2}
\end{aligned}
$$

Case (i). Due to $u_{r}, u_{z} \in B M O^{-1}\left(R^{3}\right)$, we write

$$
u_{r}=\partial_{j} d_{1, j}, \quad u_{z}=\partial_{j} d_{2, j}, \quad j=1,2,3,
$$

where $d_{1, j}, d_{2, j} \in B M O\left(R^{3}\right)$. Also, denote $\bar{f}$ as the mean value of $f$ on the domain $C_{R}$. Then we have

$$
\begin{aligned}
I= & \int_{C_{R}}\left(\frac{1}{2}|u|^{2}+p\right)\left[\partial_{j}\left(d_{1, j}-\bar{d}_{1, j}\right) \partial_{r}+\partial_{j}\left(d_{2, j}-\bar{d}_{2, j}\right) \partial_{z}\right] \phi d x \\
= & -\int_{C_{R}} \partial_{j}\left(\frac{1}{2}|u|^{2}+p\right)\left[\left(d_{1, j}-\bar{d}_{1, j}\right) \partial_{r} \phi+\left(d_{2, j}-\bar{d}_{2, j}\right) \partial_{z} \phi\right] d x \\
& -\int_{C_{R}}\left(\frac{1}{2}|u|^{2}+p\right)\left[\left(d_{1, j}-\bar{d}_{1, j}\right) \partial_{j}\left(\partial_{r} \phi\right)+\left(d_{2, j}-\bar{d}_{2, j}\right) \partial_{j}\left(\partial_{z} \phi\right)\right] d x
\end{aligned}
$$

Recall that $\phi(x)=\phi(r, z)$ and

$$
\begin{aligned}
& \partial_{j} \partial_{z} \phi=\partial_{z} \partial_{j} \phi, \quad \text { for } \quad j=1,2,3, \\
& \partial_{j} \partial_{r} \phi=\partial_{z} \partial_{j} \phi, \quad \text { for } \quad j=3, \\
& \partial_{1} \partial_{r} \phi=\cos \theta \partial_{r}^{2} \phi, \quad \partial_{2} \partial_{r} \phi=\sin \theta \partial_{r}^{2} \phi
\end{aligned}
$$

which and the property of BMO function yield that

$$
\begin{aligned}
I \leq & C R^{-1}\left\|\left|\nabla\left(|u|^{2}\right)\right|+|\nabla p|\right\|_{L^{\frac{3}{2}}\left(C_{R} \backslash C_{R / 2}\right)}\left(\left\|d_{1, j}-\bar{d}_{1, j}\right\|_{L^{3}\left(C_{R}\right)}+\left\|d_{2, j}-\bar{d}_{2, j}\right\|_{L^{3}\left(C_{R}\right)}\right) \\
& +C R^{-2}\left(\|u\|_{L^{6}\left(C_{R} \backslash C_{R / 2}\right)}^{2}+\|p\|_{L^{3}\left(C_{R} \backslash C_{R / 2}\right)}\right)\left(\left\|d_{1, j}-\bar{d}_{1, j}\right\|_{L^{\frac{3}{2}}\left(C_{R}\right)}+\left\|d_{2, j}-\bar{d}_{2, j}\right\|_{L^{\frac{3}{2}\left(C_{R}\right)}}\right) \\
\leq & C\left\|\left|\nabla\left(|u|^{2}\right)\right|+|\nabla p|\right\|_{L^{\frac{3}{2}}\left(C_{R} \backslash C_{R / 2}\right)}+C\left(\|u\|_{L^{6}\left(C_{R} \backslash C_{R / 2}\right)}^{2}+\|p\|_{L^{3}\left(C_{R} \backslash C_{R / 2}\right)}\right) \\
& \rightarrow 0 \quad(\text { as } \mathrm{R} \rightarrow \infty)
\end{aligned}
$$

Hence, the proof of case (i) is complete.

Case (ii). When $\left|\left(u_{r}, u_{z}\right)\right| \leq \frac{C}{r}$ for $r>0$,

$$
I=\int_{C_{R}}\left(\frac{1}{2}|u|^{2}+p\right)\left(u_{r} \partial_{r}+u_{z} \partial_{z}\right) \phi d x
$$




$$
\leq C \int_{C_{R}}\left(\frac{1}{2}|u|^{2}+|p|\right)\left(\partial_{r} \ln (r)\left|\partial_{r} \phi\right|+\partial_{r} \ln (r)\left|\partial_{z} \phi\right|\right) d x .
$$

Let $g(r)=\ln (r)$ and $\bar{g}$ be the mean value of $g$ on $\left\{x^{\prime} ;\left|x^{\prime}\right| \leq R\right\}$. Then we have

$$
\begin{aligned}
I \leq & -C \int_{C_{R}} \partial_{r}\left(\frac{1}{2}|u|^{2}+|p|\right)(g-\bar{g})\left(\left|\partial_{r} \phi\right|+\left|\partial_{z} \phi\right|\right) d x \\
& -C \int_{C_{R}}\left(\frac{1}{2}|u|^{2}+|p|\right)(g-\bar{g}) \partial_{r}\left(\left|\partial_{r} \phi\right|+\left|\partial_{z} \phi\right|\right) d x \\
& -C \int_{C_{R}}\left(\frac{1}{2}|u|^{2}+|p|\right)(g-\bar{g}) \frac{1}{r}\left(\left|\partial_{r} \phi\right|+\left|\partial_{z} \phi\right|\right) d x \\
& \doteq I_{1}+I_{2}+I_{3}
\end{aligned}
$$

Note that $g \in B M O\left(R^{2}\right)$ (see, for example, Chapter IV [15]), and we have

$$
R^{-1}\left(\int_{C_{R}}|g-\bar{g}|^{3} d x\right)^{\frac{1}{3}} \leq C\left(R^{-2} \int_{\left|x^{\prime}\right| \leq R}|g-\bar{g}|^{3} d x\right)^{\frac{1}{3}} \leq C
$$

and

$$
R^{-2}\left(\int_{C_{R}}|g-\bar{g}|^{\frac{2}{3}} d x\right)^{\frac{2}{3}} \leq C, \quad R^{-3}\left(\int_{C_{R}}|g-\bar{g}|^{12} d x\right) \leq C
$$

Hence as the arguments of (i), we have

$$
I_{1}+I_{2} \leq C\left\|\left|\nabla\left(|u|^{2}\right)\right|+|\nabla p|\right\|_{L^{\frac{3}{2}}\left(C_{R} \backslash C_{R / 2}\right)}+C\left(\|u\|_{L^{6}\left(C_{R} \backslash C_{R / 2}\right)}^{2}+\|p\|_{L^{3}\left(C_{R} \backslash C_{R / 2}\right)}\right)
$$

For the term of $I_{3}$, we get

$$
\begin{aligned}
I_{3} & \leq C R^{-1}\left(\|u\|_{L^{6}\left(C_{R} \backslash C_{R / 2}\right)}^{2}+\|p\|_{L^{3}\left(C_{R} \backslash C_{R / 2}\right)}\right)\|g-\bar{g}\|_{L^{12}\left(C_{R}\right)}\left\|\frac{1}{r}\right\|_{L^{\frac{12}{7}}\left(C_{R}\right)} \\
& \leq C R^{-\frac{1}{4}}\left(\|u\|_{L^{6}\left(C_{R} \backslash C_{R / 2}\right)}^{2}+\|p\|_{L^{3}\left(C_{R} \backslash C_{R / 2}\right)}\right)\|g-\bar{g}\|_{L^{12}\left(C_{R}\right)} \\
& \leq C\left(\|u\|_{L^{6}\left(C_{R} \backslash C_{R / 2}\right)}^{2}+\|p\|_{L^{3}\left(C_{R} \backslash C_{R / 2}\right)}\right)
\end{aligned}
$$

Hence, we can conclude that

$$
I \rightarrow 0 \quad(\text { as } \mathrm{R} \rightarrow \infty)
$$

The proof of Theorem 1.2 is complete.

\section{Proof of Theorem 1.3}

We are going to prove that 
Proposition 4.1. Assume that the conditions of Theorem 1.3 hold. (1) Let $w_{\theta} \leq \mathrm{Cr}^{-\beta}$ with $\beta>1$. Then we get for $r>1$

$$
\left|u_{r}(r, z)\right|+\left|u_{z}(r, z)\right| \leq C\left\{\begin{array}{l}
(1+r)^{-\frac{3}{2}+\frac{1}{2(\beta-1)}}, \quad \beta>2, \\
(1+r)^{1-\beta}, \quad 1<\beta<2, \\
(1+r)^{-1} \ln (r+1), \quad \beta=2 .
\end{array}\right.
$$

(2) Let $\left|w_{r}\right|+\left|w_{z}\right| \leq C r^{-\beta}$ with $\beta>1$. Then we get for $r>1$

$$
\left|u_{\theta}(r, z)\right| \leq C\left\{\begin{array}{l}
(1+r)^{-\frac{3}{2}+\frac{1}{2(\beta-1)}}, \quad \beta>2, \\
(1+r)^{1-\beta}, \quad 1<\beta<2, \\
(1+r)^{-1} \ln (r+1), \quad \beta=2 .
\end{array}\right.
$$

Proof of Theorem 1.3. It follows from Proposition 4.1 and Theorem 1.1 directly.

Next we are aimed to prove Proposition 4.1. Firstly, we introduce a representation formula of $u_{r}, u_{z}$ and $u_{\theta}$ with the help of the vorticity. Since $b=u_{r} e_{r}+u_{z} e_{z}$ and

$$
\nabla \times b=w_{\theta} e_{\theta}, \quad \nabla \times\left(u_{\theta} e_{\theta}\right)=w_{r} e_{r}+w_{z} e_{z}
$$

by Biot-Savart law, we can get the integral representation of the velocity as follows(for example, see Lemma 2.2 for a local version by Choe-Jin [6], also see Lemma 3.10 by Weng $[17])$.

Lemma 4.2. Like the vorticity at the point $(r \cos \theta, r \sin \theta, z)$ denoted by $\left(w_{r}, w_{\theta}, w_{z}\right)(r, z)$, we write the vorticity at the point $(\rho \cos \phi, \rho \sin \phi, k)$ as $\left(w_{\rho}, w_{\phi}, w_{k}\right)(\rho, k)$. Then we have

$$
\begin{aligned}
u_{r}(r, z)= & \int_{-\infty}^{\infty} \int_{0}^{\infty} \Gamma_{1}(r, \rho, z-k) w_{\phi}(\rho, k) \rho d \rho d k \\
u_{z}(r, z)= & -\int_{-\infty}^{\infty} \int_{0}^{\infty} \Gamma_{2}(r, \rho, z-k) w_{\phi}(\rho, k) \rho d \rho d k \\
u_{\theta}(r, z)= & \int_{-\infty}^{\infty} \int_{0}^{\infty} \Gamma_{3}(r, \rho, z-k) w_{k}(\rho, k) \rho d \rho d k \\
& -\int_{-\infty}^{\infty} \int_{0}^{\infty} \Gamma_{1}(r, \rho, z-k) w_{\rho}(\rho, k) \rho d \rho d k
\end{aligned}
$$

where

$$
\begin{aligned}
& \Gamma_{1}(r, \rho, z-k)=\frac{1}{4 \pi} \int_{0}^{2 \pi} \frac{z-k}{\left[r^{2}+\rho^{2}-2 r \rho \cos \phi+(z-k)^{2}\right]^{\frac{3}{2}}} \cos \phi d \phi \\
& \Gamma_{2}(r, \rho, z-k)=-\frac{1}{4 \pi} \int_{0}^{2 \pi} \frac{\rho-r \cos \phi}{\left[r^{2}+\rho^{2}-2 r \rho \cos \phi+(z-k)^{2}\right]^{\frac{3}{2}}} d \phi \\
& \Gamma_{3}(r, \rho, z-k)=-\frac{1}{4 \pi} \int_{0}^{2 \pi} \frac{\rho-r \cos \phi}{\left[r^{2}+\rho^{2}-2 r \rho \cos \phi+(z-k)^{2}\right]^{\frac{3}{2}}} \cos \phi d \phi .
\end{aligned}
$$


Secondly, we give the bounds of estimate of $\Gamma_{2}, \Gamma_{3}$ and $\Gamma_{1}$, which will be used in the proof. This is similar to that in [6], where $\rho \approx r$ was assumed. Here we consider all $\rho>0$ and large $r>0$. In details, we have the following estimates.

Lemma 4.3 (Estimate of $\Gamma_{2}, \Gamma_{3}$ and $\Gamma_{1}$ ).

$$
\left|\Gamma_{2}(r, \rho, z-k)\right|+\left|\Gamma_{3}(r, \rho, z-k)\right| \leq \frac{C}{(\max \{\rho, r\})^{\alpha}\left[(r-\rho)^{2}+(z-k)^{2}\right]^{\frac{2-\alpha}{2}}},
$$

for $r>1$ and $0 \leq \alpha \leq 1$;

$$
\left|\Gamma_{1}(r, \rho, z-k)\right| \leq \frac{C|z-k|}{(\max \{\rho, r\})^{\alpha}\left[(r-\rho)^{2}+(z-k)^{2}\right]^{\frac{3-\alpha}{2}}},
$$

where $r>1,0 \leq \alpha \leq 1$ for $\frac{r}{4} \leq \rho \leq 4 r$, and $0 \leq \alpha \leq 3$ for $\rho<\frac{r}{4}$ or $\rho \geq 4$.

Thirdly, we assume Lemma 4.3 holds and complete the proof of Proposition 4.1 and Lemma 4.3 is proved later.

Proof of Proposition 4.1: At first, we estimate the term of $u_{r}(r, z)$. Let

$$
\begin{aligned}
I= & u_{r}(r, z)=\int_{-\infty}^{\infty} \int_{0}^{\infty} \Gamma_{1} w_{\phi} \rho d \rho d k \\
= & \int_{-\infty}^{\infty} \int_{0}^{r^{\gamma} / 8} \Gamma_{1} w_{\phi} \rho d \rho d k+\int_{-\infty}^{\infty} \int_{r^{\gamma} / 8}^{r / 4} \Gamma_{1} w_{\phi} \rho d \rho d k+\int_{-\infty}^{\infty} \int_{r / 4}^{r-r^{\delta} / 2} \Gamma_{1} w_{\phi} \rho d \rho d k \\
& +\int_{-\infty}^{\infty} \int_{r-r^{\delta} / 2}^{r+r^{\delta} / 2} \Gamma_{1} w_{\phi} \rho d \rho d k+\int_{-\infty}^{\infty} \int_{r+r^{\delta} / 2}^{4 r} \Gamma_{1} w_{\phi} \rho d \rho d k+\int_{-\infty}^{\infty} \int_{4 r}^{\infty} \Gamma_{1} w_{\phi} \rho d \rho d k \\
= & I_{1}+\cdots+I_{6},
\end{aligned}
$$

where $0 \leq \gamma, \delta \leq 1$, to be decided.

For the term $I_{1}$, by $(12)$ and $\left\|w_{\phi}\right\|_{L^{2}\left(R^{3}\right)}^{2} \leq C D(u)<\infty$ we get

$$
\begin{aligned}
I_{1} & \leq C\left(\int_{-\infty}^{\infty} \int_{0}^{r^{\gamma} / 8}\left|\Gamma_{1}(r, \rho, z-k)\right|^{2} \rho d \rho d k\right)^{\frac{1}{2}} \\
& \leq C\left(\int_{-\infty}^{\infty} \int_{0}^{r^{\gamma} / 8} \frac{|z-k|^{2}}{r^{2 \alpha}\left[r^{2}+(z-k)^{2}\right]^{3-\alpha}} \rho d \rho d k\right)^{\frac{1}{2}} \\
& \leq C r^{-\frac{3}{2}}\left(\int_{-\infty}^{\infty} \int_{0}^{r^{\gamma} / 8} \frac{r^{-2}|z-k|^{2}}{\left[1+r^{-2}(z-k)^{2}\right]^{3-\alpha}} r^{-1} d k \rho d \rho\right)^{\frac{1}{2}} \leq C r^{-\frac{3}{2}+\gamma}
\end{aligned}
$$

where $0 \leq \alpha<\frac{3}{2}$.

For the term $I_{2}$, using $r>1,(12)$ and $w_{\theta} \leq \mathrm{Cr}^{-\beta}$

$$
I_{2} \leq C \int_{-\infty}^{\infty} \int_{r^{\gamma} / 8}^{r / 4} \Gamma_{1} \rho^{1-\beta} d \rho d k
$$




$$
\begin{aligned}
& \leq C\left(\int_{-\infty}^{\infty} \int_{r^{\gamma} / 8}^{r / 4} \frac{|z-k|}{r^{\alpha}\left[r^{2}+(z-k)^{2}\right]^{\frac{3-\alpha}{2}}} \rho^{1-\beta} d \rho d k\right) \\
& \leq C\left\{\begin{array}{l}
r^{-1+\gamma(2-\beta)} \quad(\beta>2) \\
r^{-1} \ln r \quad(\beta=2) \\
r^{1-\beta} \quad(1<\beta<2)
\end{array}\right.
\end{aligned}
$$

where $0 \leq \alpha<1$.

Moreover, for the term $I_{3}$, by (12) and $w_{\theta} \leq \mathrm{Cr}^{-\beta}$

$$
\begin{aligned}
I_{3} & \leq C \int_{-\infty}^{\infty} \int_{r / 4}^{r-r^{\delta} / 2} \Gamma_{1} \rho^{1-\beta} d \rho d k \\
& \leq C\left(\int_{-\infty}^{\infty} \int_{r / 4}^{r-r^{\delta} / 2} \frac{|z-k|}{r^{\alpha}\left[(r-\rho)^{2}+(z-k)^{2}\right]^{\frac{3-\alpha}{2}}} \rho^{1-\beta} d \rho d k\right) \\
& \leq C r^{-\alpha-\delta+\alpha \delta}\left(\int_{-\infty}^{\infty} \int_{r / 4}^{r-r^{\delta} / 2} \frac{r^{-\delta}|z-k|}{\left[\frac{1}{4}+r^{-2 \delta}(z-k)^{2}\right]^{\frac{3-\alpha}{2}}} r^{-\delta} d k \rho^{1-\beta} d \rho\right) \\
& \leq C \begin{cases}r^{2-\beta-\alpha-\delta+\delta \alpha} & (\beta<2 \text { or } \beta>2) \\
r^{-\alpha-\delta+\alpha \delta} \ln r \quad(\beta=2)\end{cases}
\end{aligned}
$$

where $0 \leq \alpha<1$.

Similarly, for $I_{5}$ we have

$$
I_{5} \leq C \begin{cases}r^{2-\beta-\alpha-\delta+\delta \alpha} & (\beta<2 \text { or } \beta>2) \\ r^{-\alpha-\delta+\alpha \delta} \ln r \quad(\beta=2)\end{cases}
$$

where $0 \leq \alpha<1$.

Furthermore, for $0 \leq \alpha<1$ by (12) and $w_{\theta} \leq \mathrm{Cr}^{-\beta}$ we have

$$
\begin{aligned}
I_{4} & \leq C \int_{-\infty}^{\infty} \int_{r-r^{\delta} / 2}^{r+r^{\delta} / 2} \Gamma_{1} \rho^{1-\beta} d \rho d k \\
& \leq C\left(\int_{-\infty}^{\infty} \int_{r-r^{\delta} / 2}^{r+r^{\delta} / 2} \frac{|z-k|}{r^{\alpha}\left[(r-\rho)^{2}+(z-k)^{2}\right]^{\frac{3-\alpha}{2}}} \rho^{1-\beta} d \rho d k\right) \\
& \leq C\left(\int_{r-r^{\delta} / 2}^{r+r^{\delta} / 2} r^{-\alpha}(r-\rho)^{-1+\alpha} \rho^{1-\beta} d \rho\right) \\
& \leq C r^{1-\beta-\alpha}\left(\int_{r-r^{\delta} / 2}^{r+r^{\delta} / 2}(r-\rho)^{-1+\alpha} d \rho\right) \\
& \leq C r^{1-\beta-\alpha+\delta \alpha}(\beta>1)
\end{aligned}
$$

Finally, (12) and $w_{\theta} \leq C r^{-\beta}$ yield that

$$
I_{6} \leq C \int_{-\infty}^{\infty} \int_{4 r}^{\infty} \Gamma_{1} \rho^{1-\beta} d \rho d k
$$




$$
\begin{aligned}
& \leq C\left(\int_{-\infty}^{\infty} \int_{4 r}^{\infty} \frac{|z-k|}{\rho^{\alpha}\left[\rho^{2}+(z-k)^{2}\right]^{\frac{3-\alpha}{2}}} \rho^{1-\beta} d \rho d k\right) \\
& \leq C r^{1-\beta}(\beta>1)
\end{aligned}
$$

Hence, concluding the estimates of $I_{1}, \cdots, I_{6}$, we have the following arguments.

Case a. $\beta>2$. At this time, we have

$$
I \leq C\left[r^{-\frac{3}{2}+\gamma}+r^{-1+\gamma(2-\beta)}+r^{2-\beta-\alpha-\delta+\delta \alpha}+r^{1-\beta-\alpha+\delta \alpha}+r^{1-\beta}\right]
$$

where $0 \leq \alpha<1$ and $0 \leq \gamma, \delta \leq 1$.

First, we choose $\gamma=\frac{1}{2(\beta-1)}$ such that $-\frac{3}{2}+\gamma=-1+\gamma(2-\beta)$. Furthermore, we take $\alpha \uparrow 1, \delta \uparrow 1$ such that

$$
(1-\delta)(1-\alpha) \leq \beta-\frac{5}{2}+\frac{1}{2(\beta-1)}
$$

which implies

$$
-1+\gamma(2-\beta) \geq 2-\beta-\alpha-\delta+\delta \alpha
$$

Moreover, note that

$$
2-\beta-\alpha-\delta+\delta \alpha \geq 1-\beta \geq 1-\beta-\alpha+\delta \alpha
$$

Then, we get for $r>1$

$$
\left|u_{r}(r, z)\right| \leq C r^{-\frac{3}{2}+\frac{1}{2(\beta-1)}}
$$

Case b. $\beta<2$. At this time, we have

$$
I \leq C\left[r^{-\frac{3}{2}+\gamma}+r^{2-\beta-\alpha-\delta+\delta \alpha}+r^{1-\beta-\alpha+\delta \alpha}+r^{1-\beta}\right]
$$

where $0 \leq \alpha<1$ and $0 \leq \gamma, \delta \leq 1$. We choose $\gamma=0$ and $\delta=1$, then we get

$$
\left|u_{r}(r, z)\right| \leq C r^{1-\beta}
$$

Case c. $\beta=2$. At this time, we have

$$
I \leq C\left[r^{-\frac{3}{2}+\gamma}+r^{-1} \ln r+r^{-\alpha-\delta+\alpha \delta} \ln r+r^{1-\beta-\alpha+\delta \alpha}+r^{1-\beta}\right]
$$

where $0 \leq \alpha<1$ and $0 \leq \gamma, \delta \leq 1$. We choose $\gamma=0$ and $\delta=1$, then we get

$$
\left|u_{r}(r, z)\right| \leq C r^{-1} \ln r
$$

Hence we complete the estimate of $u_{r}(r, z)$. 
Note that the bound of $\Gamma_{1}$ used as above is similar to the estimates of $\Gamma_{2}$ and $\Gamma_{3}$. Hence similar arguments hold for $u_{z}$ and $u_{\theta}$. The proof of Proposition 4.1 is complete.

Proof of Lemma 4.3. The remaining part is devoted to proving Lemma 4.3, which is similar to that of [6], where the case $\frac{r}{4}<\rho<4 r$ is discussed. Here we consider all the value $\rho>0$ and sketch the proof. First, for $k>0$ and $\beta \geq 1$ we find

$$
I=\int_{0}^{\frac{\pi}{2}} \frac{d \phi}{\left(\sqrt{1+k \sin ^{2} \phi}\right)^{\beta}} \leq \begin{cases}C(\delta) \min \left\{1, k^{-\frac{\delta}{2}}\right\}, & \beta=1 \\ C(\beta) \min \left\{1, k^{-\frac{1}{2}}\right\}, & \beta>1\end{cases}
$$

for any $0 \leq \delta<1$. Obviously, $k \leq C$ holds, and next we assume that $k$ is large enough. Then for $0<\ell<1$

$$
I \leq \ell+\int_{\ell}^{\frac{\pi}{2}} \frac{d \phi}{\left(k \sin ^{2} \phi\right)^{\beta / 2}}
$$

Due to $\phi \leq 2 \sin \phi$ for $\phi \in\left(0, \frac{\pi}{2}\right)$, we have

$$
I \leq \ell+2 k^{-\beta / 2}\left(\ln \left(\frac{\pi}{2}\right)-\ln \ell\right), \quad \beta=1
$$

and

$$
I \leq \ell+2^{\beta} k^{-\beta / 2} \frac{\left(\frac{\pi}{2}\right)^{1-\beta}-\ell^{1-\beta}}{1-\beta}, \quad \beta>1,
$$

which yield the required bound (13) by choosing a suitable $\ell$.

Obviously, from the formulas of $\Gamma_{2}, \Gamma_{3}$ and $\Gamma_{1}$, we have

$$
\begin{gathered}
\left|\Gamma_{i}(r, \rho, z-k)\right| \leq \frac{\rho+r}{\left[(r-\rho)^{2}+(z-k)^{2}\right]^{\frac{3}{2}}}, \quad i=2,3 \\
\left|\Gamma_{1}(r, \rho, z-k)\right| \leq \frac{|z-k|}{\left[(r-\rho)^{2}+(z-k)^{2}\right]^{\frac{3}{2}}}
\end{gathered}
$$

for all $\rho>0$ and $r>0$.

Next we go on estimating $\Gamma_{2}, \Gamma_{3}$, and $\Gamma_{1}$ carefully, respectively.

Step I. Noting the periodic and even property and variable transform for $\phi$, we also have

$$
\begin{aligned}
\Gamma_{2} & =-\int_{0}^{2 \pi} \frac{1}{4 \pi} \frac{\rho-r \cos \phi}{\left[r^{2}+\rho^{2}-2 r \rho \cos \phi+(z-k)^{2}\right]^{\frac{3}{2}}} d \phi \\
& =-\int_{0}^{\frac{\pi}{2}} \frac{1}{\pi} \frac{\rho-r \cos 2 \phi}{\left[r^{2}+\rho^{2}-2 r \rho \cos 2 \phi+(z-k)^{2}\right]^{\frac{3}{2}}} d \phi
\end{aligned}
$$

and

$$
\Gamma_{2}=-\int_{0}^{\frac{\pi}{2}} \frac{1}{2 \pi} \frac{\rho^{2}-2 r \rho \cos 2 \phi+r^{2}+\rho^{2}-r^{2}}{\rho\left[(r-\rho)^{2}+4 r \rho \sin ^{2} \phi+(z-k)^{2}\right]^{\frac{3}{2}}} d \phi
$$




$$
\begin{aligned}
\leq & C \frac{1}{\rho \sqrt{(r-\rho)^{2}+(z-k)^{2}}} \int_{0}^{\pi / 2} \frac{d \phi}{\sqrt{1+K \sin ^{2} \phi}} \\
& -\frac{1}{2 \pi} \frac{1}{\rho\left[(r-\rho)^{2}+(z-k)^{2}\right]^{\frac{3}{2}}} \int_{0}^{\pi / 2} \frac{\rho^{2}-r^{2}}{\left(\sqrt{1+K \sin ^{2} \phi}\right)^{3}} d \phi \\
\doteq & I_{1}+I_{2}
\end{aligned}
$$

where

$$
K=\frac{4 r \rho}{(r-\rho)^{2}+(z-k)^{2}}
$$

When $K \leq 1$, that is $4 r \rho \leq(r-\rho)^{2}+(z-k)^{2}$, we have $(r-\rho)^{2}+(z-k)^{2} \geq \frac{1}{2} r^{2}$ for $\rho \leq \frac{r}{2}$ and $(r-\rho)^{2}+(z-k)^{2} \geq 2 r^{2}$ for $\frac{r}{2} \leq \rho \leq 4 r$. Moreover, for $\rho \geq 4 r$ we have

$$
(r-\rho)^{2}+(z-k)^{2} \geq\left(\frac{3}{4} \rho\right)^{2} \geq\left(\frac{3}{5}(\rho+r)\right)^{2} \geq \frac{9}{25}(\rho+r)^{2}
$$

Hence for $K \leq 1$ we have

$$
\Gamma_{2} \leq C \frac{1}{\rho \sqrt{(r-\rho)^{2}+(z-k)^{2}}}
$$

When $K>1$, by (13) we have

$$
\begin{aligned}
\Gamma_{2} \leq & C(\delta) \frac{1}{\rho \sqrt{(r-\rho)^{2}+(z-k)^{2}}} \\
\cdot & {\left[\left(\frac{(r-\rho)^{2}+(z-k)^{2}}{4 r \rho}\right)^{\frac{\delta}{2}}+\frac{\left|\rho^{2}-r^{2}\right|}{(r-\rho)^{2}+(z-k)^{2}}\left(\frac{(r-\rho)^{2}+(z-k)^{2}}{4 r \rho}\right)^{\frac{1}{2}}\right] }
\end{aligned}
$$

where $0 \leq \delta<1$.

Case a. For $r>1$ and $\rho \leq \frac{r}{4}$ or $\rho>4 r$, by (14) we know the estimate (11) holds.

Case b. For $r>1$ and $\frac{r}{4} \leq \rho \leq 4 r$ with $K \leq 1$, by (14) and (16) we know the estimate (11) holds.

Case c. For $r>1$ and $\frac{r}{4} \leq \rho \leq 4 r$ with $K>>1$, by (14) and (17) we know the estimate (11) holds by noting that $(r-\rho)^{2}+(z-k)^{2} \leq 16 r^{2}$ and

$$
\frac{\left|\rho^{2}-r^{2}\right|}{(r-\rho)^{2}+(z-k)^{2}}\left(\frac{(r-\rho)^{2}+(z-k)^{2}}{4 r \rho}\right)^{\frac{1}{2}} \leq \frac{\rho+r}{\sqrt{4 r \rho}} \leq 5 .
$$

Hence the proof of $\Gamma_{2}$ is complete.

Step II. The term $\Gamma_{2}$ is similar and we omitted the details.

Step III. The term $\Gamma_{1}$ is estimated as follows.

$$
\Gamma_{1}(r, \rho, z-k)=\frac{1}{2 \pi} \int_{0}^{\pi} \frac{z-k}{\left[r^{2}+\rho^{2}-2 r \rho \cos \phi+(z-k)^{2}\right]^{\frac{3}{2}}} \cos \phi d \phi
$$




$$
\begin{aligned}
& =\frac{1}{\pi} \int_{0}^{\frac{\pi}{2}} \frac{z-k}{\left[(r-\rho)^{2}+4 r \rho \sin ^{2} \phi+(z-k)^{2}\right]^{\frac{3}{2}}} \cos 2 \phi d \phi \\
& \leq C \frac{|z-k|}{\left[(r-\rho)^{2}+(z-k)^{2}\right]^{\frac{3}{2}}} \int_{0}^{\pi / 2} \frac{1}{\left(\sqrt{1+K \sin ^{2} \phi}\right)^{3}} d \phi \\
& \doteq I^{\prime}
\end{aligned}
$$

where

$$
K=\frac{4 r \rho}{(r-\rho)^{2}+(z-k)^{2}}
$$

When $K \leq 1$, i.e. $4 r \rho \leq(r-\rho)^{2}+(z-k)^{2}$, we have $(r-\rho)^{2}+(z-k)^{2} \geq \frac{1}{2} r^{2}$ for $\rho \leq \frac{r}{2}$ and $(r-\rho)^{2}+(z-k)^{2} \geq 2 r^{2}$ for $\frac{r}{2} \leq \rho \leq 4 r$. Moreover, for $\rho \geq 4 r$ we have

$$
(r-\rho)^{2}+(z-k)^{2} \geq\left(\frac{3}{4} \rho\right)^{2}
$$

Hence for $K \leq 1$ we have

$$
(r-\rho)^{2}+(z-k)^{2} \geq \frac{1}{2}(\max \{r, \rho\})^{2}
$$

Using (15), for $K \leq 1$ we get

$$
\left|\Gamma_{1}(r, \rho, z-k)\right| \leq \frac{C|z-k|}{(\max \{\rho, r\})^{\alpha}\left[(r-\rho)^{2}+(z-k)^{2}\right]^{\frac{3-\alpha}{2}}},
$$

where $0 \leq \alpha \leq 3$.

When $K>1$, i.e. $4 r \rho \geq(r-\rho)^{2}+(z-k)^{2}$, which implies $\rho>\frac{1}{8} r$, by (13) we have

$$
\begin{aligned}
\left|\Gamma_{1}(r, \rho, z-k)\right| & \leq \frac{C|z-k|}{\left[(r-\rho)^{2}+(z-k)^{2}\right]^{\frac{3}{2}}}\left(\frac{(r-\rho)^{2}+(z-k)^{2}}{4 r \rho}\right)^{\frac{1}{2}} \\
& \leq \frac{C|z-k|}{\sqrt{r \rho}\left[(r-\rho)^{2}+(z-k)^{2}\right]}
\end{aligned}
$$

Thus for $\frac{1}{8} r<\rho<4 r$, we have

$$
\left|\Gamma_{1}(r, \rho, z-k)\right| \leq \frac{C|z-k|}{(\max \{\rho, r\})^{\alpha}\left[(r-\rho)^{2}+(z-k)^{2}\right]^{\frac{3-\alpha}{2}}}
$$

where $0 \leq \alpha \leq 1$. For $\rho \geq 4 r$, by (15) we also derive that

$$
\left|\Gamma_{1}(r, \rho, z-k)\right| \leq \frac{C|z-k|}{(\max \{\rho, r\})^{\alpha}\left[(r-\rho)^{2}+(z-k)^{2}\right]^{\frac{3-\alpha}{2}}}
$$

where $0 \leq \alpha \leq 3$.

Concluding the estimates (18), (19) and (20), we complete the proof of the inequality (12).

Acknowledgments. W. Wang was supported by NSFC under grant 11671067, "the Fundamental Research Funds for the Central Universities" and China Scholarship Council. 


\section{References}

[1] B. Carrillo, X. Pan, Q. Zhang, Decay and vanishing of some axially symmetric D-solutions of the Navier-Stokes equations, arXiv:1801.07420 [math.AP].

[2] D. Chae, Liouville-type theorem for the forced Euler equations and the NavierStokes equations. Commun. Math. Phys., 326: 37-48 (2014).

[3] D. Chae, T. Yoneda, On the Liouville theorem for the stationary Navier-Stokes equations in a critical space, J. Math. Anal. Appl. 405 (2013), no. 2, 706-710.

[4] D. Chae, J. Wolf, On Liouville type theorems for the steady Navier- Stokes equations in $R^{3}$, arXiv:1604.07643.

[5] Chae, G., Weng, S., Liouville type theorems for the steady axially symmetric Navier-Stokes and Magnetohydrodynamic equations, Discrete and Continuous Dynamical Systems, Volume 36, Number 10, 2016, 5267-5285.

[6] H. Choe, B.Jin, Asymptotic properties of axis-symmetric D-solutions of the Navier-Stokes equations. J. Math. Fluid Mech. 11 (2009), no. 2, 208-232.

[7] G. P. Galdi, An introduction to the mathematical theory of the Navier-Stokes equations. Steady-state problems. Second edition. Springer Monographs in Mathematics. Springer, New York, 2011. xiv+1018 pp.

[8] Grafakos, Loukas, Classical Fourier analysis, Graduate Texts in Mathematics, 249 (2nd ed.), Berlin, New York: Springer-Verlag, 2008. doi:10.1007/978-0-38709432-8, ISBN 978-0-387-09431-1, MR 2445437.

[9] Koch, G., Nadirashvili, N., Seregin, G., Sverak, V., Liouville theorems for the Navier-Stokes equations and applications, Acta Mathematica, 203 (2009), 83105.

[10] H. Kozono, Y. Terasawab, Y. Wakasugib, A remark on Liouville-type theorems for the stationary Navier-Stokes equations in three space dimensions, Journal of Functional Analysis, 272(2017), 804-818.

[11] M. Korobkov, K. Pileckas and R. Russo, The Liouville theorem for the steadystate Navier-Stokes problem for axially symmetric 3D solutions in absence of swirl, J. Math. Fluid Mech., 17 (2015), 287-293.

[12] G. Seregin, Liouville type theorem for stationary Navier-Stokes equations, Nonlinearity, 29 (2016), 2191-2195. 
[13] G. Seregin, A liouville type theorem for steady-state Navier-Stokes equations, arXiv:1611.01563 and J. E. D. P. (2016), Expos no IX, 5 p.

[14] G. Seregin, Remarks on Liouville type theorems for steady-state Navier-Stokes equations, arXiv:1703.10822v1 and Algebra i Analiz, 2018, Vol. 30, no.2., 238248.

[15] E.M. Stein, Harmonic analysis: real-variable methods, orthogonality, and oscillatory integrals, Princeton University Press, Princeton, New Jersey, 1993.

[16] G. Seregin, W. Wang, Sufficient conditions on Liouville type theorems for the 3 steady Navier-Stokes equations, arXiv:1805.02227v1

[17] S. Weng, Decay properties of axially symmetric D-solutions to the steady NavierStokes equations, J. Math. Fluid Mech. (2017). DOI 10.1007/s00021-016-0310-5 